\title{
Toy Monte-Carlo simulation of the OLVE-HERO detector
}

I. Satyshev $^{a, 1^{*}}$, A.Pan $^{a, b}$ and L.G.Tkachev ${ }^{a, c}$

${ }^{a}$ Joint Institute for Nuclear Research, Dubna,

141980, Dubna, Russia

${ }^{b}$ The Institute of Nuclear Physics, Ministry of Energy of the Republic of Kazakhstan,

050032, Almaty, Kazakhstan

${ }^{c}$ Dubna State University,

141980, Dubna, Russia

E-mail: satyshevi@gmail.com

\begin{abstract}
A project of the OLVE-HERO space detector is proposed for CR measurement in the range $10^{12}-10^{16} \mathrm{eV}$, which will include a large ionization-neutron 3D calorimeter with a high granularity and geometric factor of $\sim 16 \mathrm{~m}^{2} \cdot \mathrm{sr}$. Current OLVE-HERO main detector is expected to be an image calorimeter of a boron loading of plastic scintillator with a tungsten absorber. Such a calorimeter allows one to measure an additional neutron signal which will improve the energy resolution of the detector and mainly the rejection power between electromagnetic and nuclear CR components will be increased by factor 30-50. The OLVE-HERO boron-loaded prototype detector was designed and tested at SPS CERN during Pb ion run in 2018. Results of the OLVE-HERO toy Monte-Carlo simulation are presented.
\end{abstract}

37th International Cosmic Ray Conference (ICRC 2021)

July 12 th $-23 r d, 2021$

Online - Berlin, Germany 


\section{Introduction}

The energy range of $10^{14}-10^{16} \mathrm{eV}$ in the classical "knee" of the galactic Cosmic Rays (CR) spectrum is of great importance for study of the mechanisms of CRs acceleration and their propagation in our Galaxy. There are no direct measurements of the CR nuclei spectra specifically in the "knee" area. Basic information about CR nuclei in the area of $10^{12}-10^{14} \mathrm{eV}$ was obtained in the balloon experiments ATIC [1,2], CREAM [3,4], TRACER[5] and on satellites AMS02 [6,7], PAMELA [8] and NUCLEON[9]. Nowadays the CR study in satellite experiments CALET [10] and DAMPE [11] have been started.

Determination of the spectrum of high-energy electrons $\left(>10^{10} \mathrm{eV}\right)$ and its possible anisotropy is important for solving the problem of the near CR sources. Extension of the secondary CR' spectra measurement by two orders of energy, i. e. CR nuclei ( $\mathrm{Li}, \mathrm{Be}, \mathrm{B}$, sub-Fe etc.), will make it possible to research the processes of CR propagation in the Galaxy in detail.

The fundamental problem of modern physics is to establish the nature of dark matter, the existence of which is firmly established from the different gravitational effects and analysis of the microwave radiation anisotropy. The search for the dark matter particles requires precise measurements of the electrons and gamma rays spectra up to the tens of $\mathrm{TeV}$.

Therefore, additional direct measurements of CR at energies up to $1000 \mathrm{TeV}$ with elemental resolution and measurements of gamma ray flux of $\mathrm{TeV}$ energies are needed. Due to the small flux of $\mathrm{CR}$, its effective measurement at such high energies requires a large geometric factor.

Within a framework of the Federal Space Research Program is proposed the High-Energy Rays Observatory (OLVE - HERO) for the research of the cosmic radiation in the energy range $10^{10}-10^{16} \mathrm{eV}$. The distinctive characteristic of this project is a high exposure factor that will enable us to resolve the above-mentioned scientific problems.

The parameters of high-energy cosmic radiation are the type of particle, the value of its kinetic energy and the arrival direction. Therefore, the proposed design of the OLVE-HERO detector is based on the development of a heavy $(\sim 10 \mathrm{t})$ ionization-neutron 3D image calorimeter with a unique high geometric factor $\left(\sim 16 \mathrm{~m}^{2} \cdot \mathrm{sr}\right)$. This geometric factor is achieved by optimizing design of the absorber and detecting elements. The three-dimensional structure of the detectors will make it possible to register particles coming from different directions. As the main detector is expected a scintillation-tungsten image calorimeter with boron loaded scintillator as an active element. This will make it possible to measure an additional signal from neutrons that would improve the energy resolution of the calorimeter, and most importantly, will increase by 30-50 times the level of rejection between the electromagnetic and hadron-nuclear components of $\mathrm{CR}$ in the whole energy range. 


\section{Simplify OLVE-HERO detector simulation}

An incoming particle interacts with a detector nucleus knocking out the secondary particles and leaving the hit nucleus in a strongly excited state. Removal of the nucleus excitation occurs due to an evaporation of $\mathrm{n}, \mathrm{p}$ and more complex nuclear fragments and $\gamma$-quanta. The average energy of evaporative neutrons is $\sim 2 \mathrm{MeV}$ and they have an isotropic angular distribution. Evaporated neutrons are slowed down to thermal energy and interact with a boron-10 nucleus to produce an $\alpha$-particle and the ${ }^{7} \mathrm{Li}$ nucleus with a total energy of $2.78 \mathrm{MeV}$.
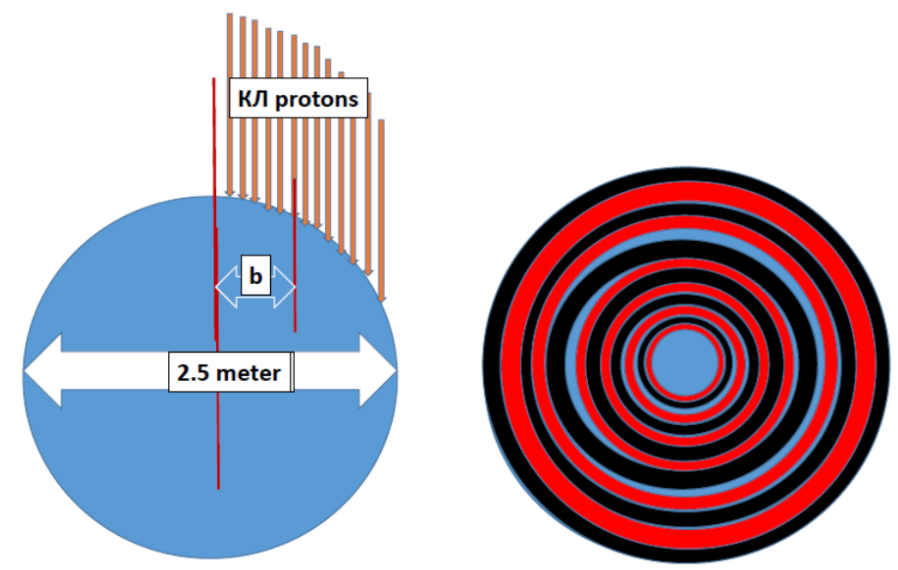

Fig. 1. Simplify schematic view of the OLVE-HERO detector

The choice of ${ }^{10} \mathrm{~B}$ is due to the large neutron capture cross section of 755 barn and an energetic $\alpha$-particle production:

$$
\mathrm{n}+{ }^{10} \mathrm{~B} \rightarrow{ }^{7} \mathrm{Li}+\alpha+\gamma+2.78 \mathrm{MeV} .
$$

The weight fraction of boron in the scintillator is $\sim 5 \%$. Most of the energy in this reaction is carried away by $\alpha$-particles $(1.47 \mathrm{MeV})$ and is spent on generating a scintillation signal. Its amplitude is equivalent to the signal from an electron with an energy of $76 \mathrm{keV}$. The typical deceleration time of an evaporative neutron with energy of $\sim 2 \mathrm{MeV}$ in polyethylene is $2.7 \mu \mathrm{s}$. The average characteristics of thermal neutrons, including the density and the spectrum $n(r, \varepsilon)$, depend on the size and configuration of the detector. The role of elastic scattering is very large, since neutron deceleration mainly occurs through elastic scattering. A propagation of the thermalized neutrons inside the detector are described according to QGSP_INCLXX+HP and https://geant4.web.cern.ch/node/155. The Monte-Carlo simulation was fulfilled for better understanding of the expected detector properties. It was carried out using the framework of software packages FairRoot and Geant4. 


\section{Results of the simulation}

It is not necessary to simulate the detailed structure of the OLVE-HERO detector to evaluate its basic characteristics such as a level of an internal unrecoverable background due to $\alpha$-particle production in the reaction (1). A simpler toy Monte-Carlo simulation was done for this purpose. Detector was considered as a spherical shape of $125 \mathrm{~cm}$ radius consisting of homogeneous Geant 4 mixture of elements: density is $2.840 \mathrm{~g} / \mathrm{cm} 3$, radiation length $3.3 \mathrm{~cm}$, nuclear interaction length $44.4 \mathrm{~cm}$, carbon, hydrogen, boron and tungsten mass fractions are in proportion $28.7 \%, 2.7 \%, 1.7 \%$ and $66,9 \%$ respectively.

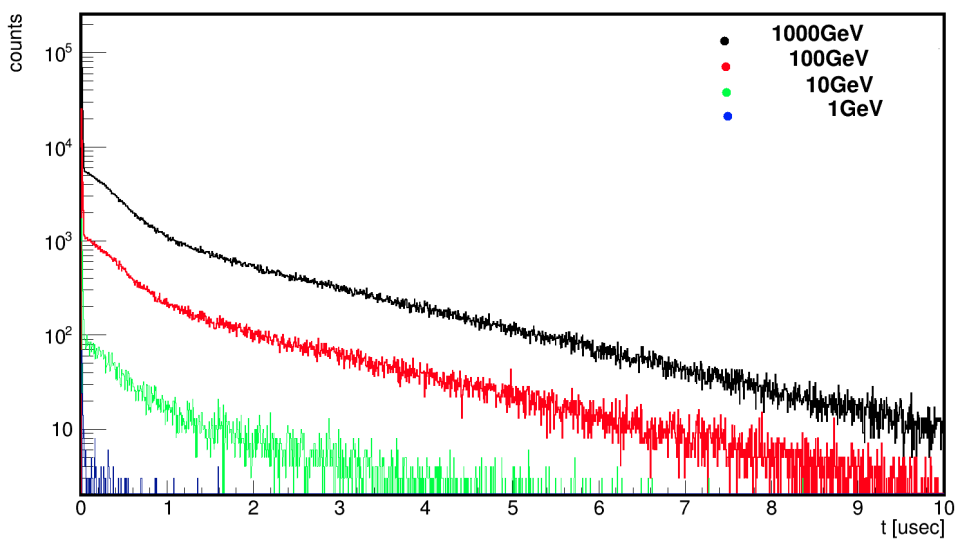

Fig. 2. The lifetime distributions of evaporated neutrons inside the detector for the different energies of incoming protons.

The evaporated neutrons are produced inside the detector, decelerated and partially absorbed according to (1) or partially escaped the detector without interaction. In Fig. 2 such lifetimes of evaporated neutrons inside the detector are presented. Two linear dependencies of the distributions are shown: a deceleration interval of the evaporated neutron with $\mathrm{t}<1 \mu$ s and a propagation interval of the thermalized neutrons with $t>1 \mu \mathrm{s}$. It is natural to expect independence of speeds of these two processes of the primary CR energies. An escape of the fast secondary neutrons is inside the first bin in Fig.2. It is also seen that the increasing of the neutron quantity for energy $\mathrm{E}=1000 \mathrm{GeV}$ is not so high in comparison with smaller initial CR energies due to more secondary cascade hadrons escaping the detector without interactions.

Important characteristics is an average lifetimes of evaporated neutrons inside the detector because it defines a time duration interval for which successive CR events signals must be accumulated. The average lifetimes of evaporated neutrons are presented in Fig.3, it is about 1 $\mu$ s and there is no clear dependence of the initial CR proton energy. The number of accumulated CR events is a very important value because it will define the number and density of the thermalized neutrons which are in equilibrium with the incoming CR flux. The internal thermalized neutron density will define a number of produced $\alpha$-particles and therefore the background noise. 


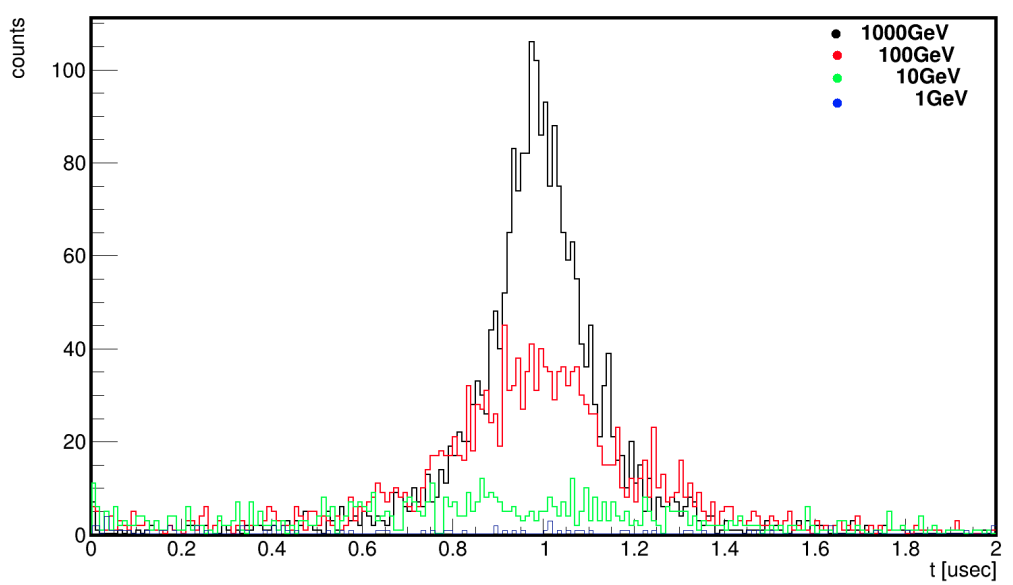

Fig. 3. The average lifetimes of thermalized neutrons inside the detector for the different energies of incoming protons

There is an additional parameter of event that is connected with a number of accumulated CR events namely the time of the last neutron escape of the detector. This can be considered as an event presence in the detector, and the corresponding distributions are presented in Fig. 4. There is clear energy dependence of this parameter - the higher the initial $\mathrm{CR}$ proton energy, the longer the event is remembered by the detector.

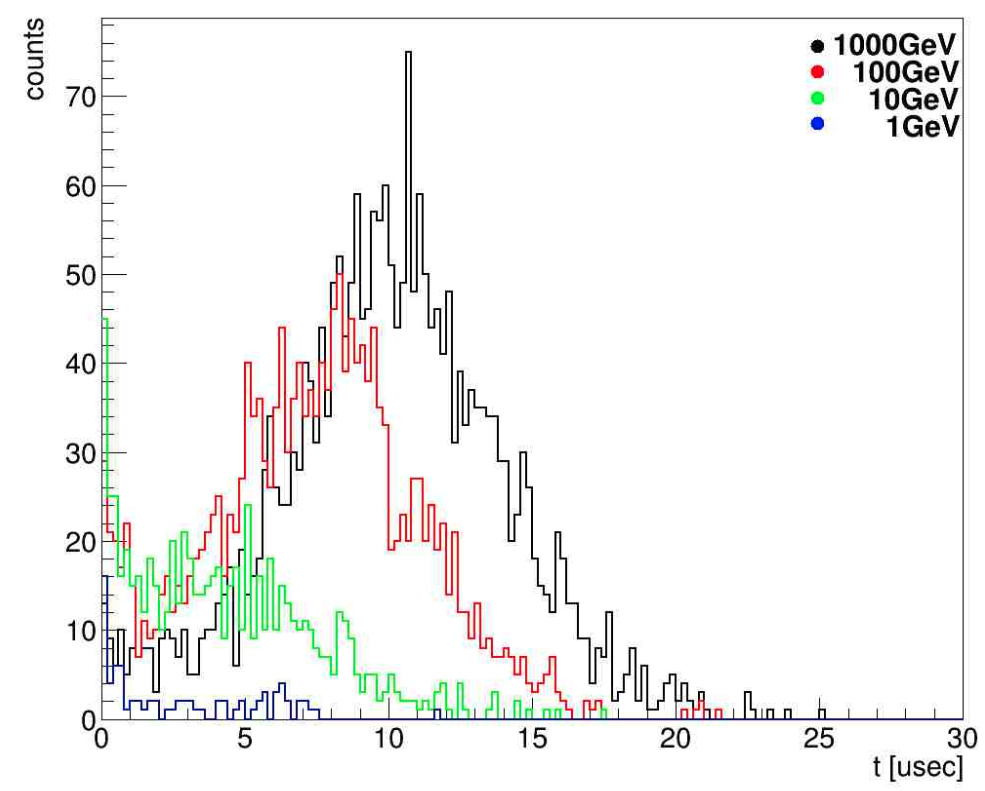

Fig. 4. The time escape distributions of the last thermalized neutrons for the different energies of incoming protons

The next natural step of the study is to use CR energy distribution for initial protons instead of a few fixed energies and to use initial particles of the different impact parameters. We generate primary protons with energies which were obtained from the known CR energy spectrum. Also, the directions of the protons were obtained from the uniform distribution, that 
they hit the detector with different uniform impact parameters. The proton source is located at a distance of $500 \mathrm{~cm}$ from the center of the detector. There was also a $\theta$ spread from $-15^{\circ}$ to $15^{\circ}$. This range was chosen based on the fact that all protons inside it fall into the detector, including those protons that fall into the detector with the maximum impact parameter.

In Fig. 5 the average escape time distribution of evaporated and thermalized neutrons when they leave the detector or are absorbed by it is presented. It is natural to assume that the first peak corresponds to evaporated neutrons which escape the detector before thermalization, and the second peak corresponds to the lifetime of the thermalized neutrons.

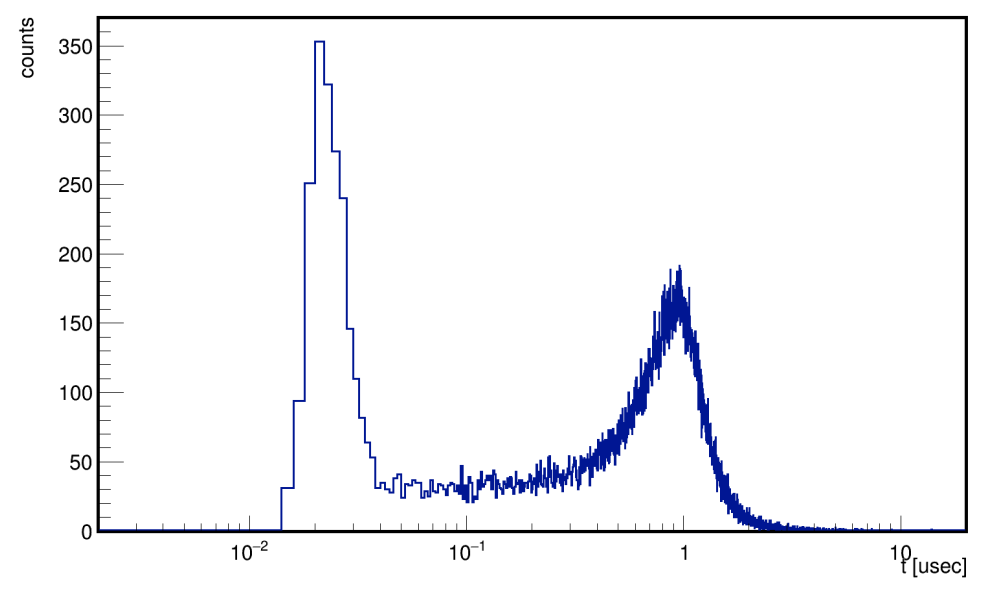

Fig. 5. The average time distribution of evaporated and thermalized neutrons, when they leave the detector or are absorbed by it.

A histogram in Fig. 6 was added because it can be plausibly interpreted. A straight line along the border region inhabited by the points corresponds to the event where the neutron disappeared simultaneously with the appearance of $\alpha$-particles $t(n)=t(\alpha)$, i.e., reaction (1). Points below this line correspond to the events of $t(n)>t(\alpha)$, i.e. the alpha particle is formed earlier as a result of the capture of another neutron.

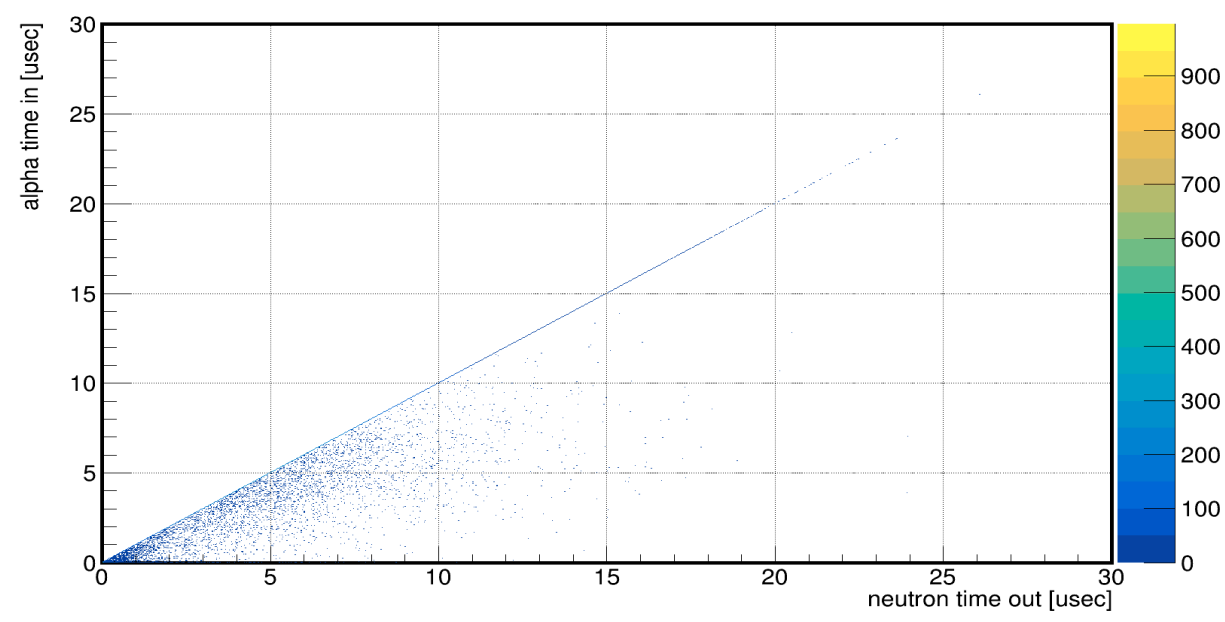

Fig. 6. The correlation for times of the last thermalized neutrons disappearance and the $\alpha$-particle appearance. 


\section{Conclusion}

The preliminary results of the simple OLVE-HERO detector simulation are presented. Using a boron-loaded scintillator as a detector and neutron moderator leads to delayed signals from the neutron capture of boron-10 after the primary interaction of the CR particle. The first significant calculations have been done, which make it possible to estimate the sensitivity of the detector in a real configuration for $\mathrm{CR}$ in the region of $10^{14-} 10^{16} \mathrm{eV}$ with taking into account the background of produced $\alpha$-particles.

The density of the CR flux in the orbit of the Earth satellite with energy of $\mathrm{E}>1 \mathrm{GeV} / \mathrm{n}$ is $\sim 1.2 \cdot 10^{8} / \mathrm{cm}^{2}$ sr.year. This flux will generate evaporation and then thermalized neutrons. Thus, inside the detector the equilibrium density of thermal neutrons is established, which determines the value of the constant background signal in the boron-loaded scintillator, which can "score" the signal from the CR showers, so there is a fear that the detector will "go blind" or give incorrect results. To obtain the final answer, it is necessary to carry out additional tests on the beams and simulation of this effect, taking into account the spectrum and composition of the CRs, as well as the geometry of the detector. Those further works are in progress.

\section{References}

[1] Ahn H.S. et al. The Energy Spectra of Protons and Helium Measured with the ATIC Experiment /Advances in Space Research. 2006. V. 37. P. 1950-1954.

[2] Panov A.D. et al. The Energy Spectra of Heavy Nuclei Measured by the ATIC Experiment. Advances in Space Research. 2006. V. 37. P. $1944-1949$.

[3] Yoon Y.S. et al. Cosmic-ray Proton and Helium Spectra from the First CREAM Flight The Astrophysical Journal. 2011. V. 728. P. 122.

[4] Ahn H.S. et al. Energy Spectra of Cosmic-ray Nuclei at High Energies. The Astrophysical Journal. 2009. V. 707. P. $593-603$.

[5] Obermeier A. et al. Energy Spectra of primary and Secondary Cosmic-ray Nuclei Measured with TRACER. The Astrophysical Journal. 2011. V. 742. P. 14.

[6] [6] Aguilar M. et al. Precision Measurement of the Helium Flux in Primary Cosmic Rays of Rigidities 1.9 GV to 3 TV with the Alpha Magnetic Spectrometer on the International Space Station // Phys. Rev. Lett. 2015. V. 115. P. 211101.

[7] [7] Aguilar M. et al. Precision Measurement of the Proton Flux in Primary Cosmic Rays from Rigidity 1 GV to 1.8 TV with the Alpha Magnetic Spectrometer on the International Space Station // Phys.Rev. Lett. 2015. V. 114. P. 171103.

[8] Adriani O. et al. // Phys. Rev. Lett. 2011. V. 106. P. 201101.

[9] Atkin, E.V. et al. Astron.Rep. 63 (2019) no.1, 66-78, Astron.Zh. 96 (2019) no.1, 75-88

[10] Brogi P, et al. CALET on the International Space Station: the first three years of observations. Phys.Scripta 95 (2020) 7, 074012.

[11] Piergiorgio Fusco for the collaboration. Main results of the DAMPE space detector after 4 years in orbit. Int.J.Mod.Phys.A 35 (2020) 36, 2044024 